\section{Mutations in L-Forms}

Conversion of bacteria into L-forms involves changes in physiology as well as morphology. Comparing sensitivity to drugs of L-forms and bacteria, we are struck by the much higher resistance of the former to penicillin. Strains of L-forms of Proteus used in our laboratory are able to grow in the presence of 60,000 units penicillin $/ \mathrm{ml}$. On the other hand, they are more sensitive to streptomycin, chlortetracycline and chloramphenicol than are the bacterial forms. Tulasne and Minck ${ }^{1}$ found that stable $\mathbf{L}$-forms of $P$. morganii were 8,000 times and unstable L-forms of $P$. vulgaris were 25,000 times more sensitive than the corresponding bacterial forms. Lederberg and St. Clair ${ }^{2}$ showed a fifty-fold decrease in resistance to streptomycin after converting Escherichia coli to the L-form. The authors suggest that "this can be viewed as the negation of the $S^{R}$ effect when the wall is stripped or a mutual reinforcement of penicillin and streptomycin in respect to another target". They were inclined to accept the former interpretation. Horoszewicz and Horoszewicz ${ }^{3}$ noted the inhibitory action of streptomycin on the conversion of $P$. mirabilis to the L-form. In the presence of the antibiotic it was necessary to inoculate 1,800 times more bacteria to obtain the same number of L-colonies.

We have used a stable L-form of Proteus mirabilis subcultured more than 100 times on serum-agar and then adapted to charcoal-agar and lately to charcoalbroth 4 . Growth of L-forms in the charcoal media was much more luxuriant than in serum media. In charcoal-broth in 2-3 days, L-organisms formed a remarkable turbidity and some days later a thick, greyish pellicle which could be dispersed on shaking. Usually the number of L-colonies grown from $1 \mathrm{ml}$. of fluid medium was $2-4 \times 10^{8}$. Such dense fluid culture was suitable for study of the formation of antibiotic-resistant mutants. Our bacterial strain was inhibited by 10 units streptomycin $/ \mathrm{ml}$. while the stabilized $\mathrm{L}$-form was sensitive to $0.0001 \mathrm{unit} / \mathrm{ml}$. For antibiotic sensitivity tests, between 100 and 1,000 organisms were used per plate. To obtain mutants resistant to streptomycin we used 4-10-day old cultures of $\mathbf{L}$-forms in charcoal-broth and added an equal volume of charcoal-broth with streptomycin. It was found that the time and frequency of mutation depended on the number of organisms in the culture. When the total number of $\mathrm{L}$-form particles was $10^{11}$, organisms resistant to 500 units streptomycin $/ \mathrm{ml}$. soon appeared. In 10 per cent of the flasks containing about $10^{9}$ organisms, mutants resistant to 50 units $/ \mathrm{ml}$. appeared first, but after 5 days it was possible to isolate mutants insensitive to $500 \mathrm{units} / \mathrm{ml}$. We did not succeed in isolating mutants resistant to 500 units/ $\mathrm{ml}$. from populations of only $3-4 \times 10^{7}$. Subculturing resistant strains on media with or without streptomycin caused no change in the character of the resistance to antibiotics.

We have also examined the streptomycin sensitivity of unstable $\mathrm{L}$-forms derived from four strains of Proteus mirabilis and from their streptomycinresistant mutants. L-forms obtained from $S^{R}$ bacteria showed higher resistance than those from $S S$ organisms. Unstable L-forms derived from streptomycinresistant bacteria could grow in the presence of 500 units streptomycin $/ \mathrm{ml}$., but we noticed that on charcoal-agar containing streptomycin but without penicillin the reversion from $\mathrm{L}$-form to bacterial form was greatly delayed in comparison with that on charcoal-agar without either antibiotic. However, after ten subcultures, the time of reversion became independent of the presence or absence of streptomycin.

L-forms insensitive to 200 units chloramphenicol/ ml., obtained by selection, did not show persistent resistance-usually after five or more passages on the medium containing chloramphenicol the culture was completely inhibited.

It was not possible to obtain antibiotic-resistant mutants by ultra-violet irradiation.

Our preliminary investigations suggest that mutants resistant to streptomycin can appear in L-form populations. It has been possible by selection to obtain mutants even more resistant to streptomycin than their corresponding bacterial forms. Probably the highly resistant mutants are formed stepwise rather than as one-step mutants.

ZB. LORKIEWICZ

IRENA ZELAZNA

Department of General Microbiology

University of Lublin, Poland.

${ }^{1}$ Tulasne, R., and Minck, R., C.R. Soc. Biol., 146 (9-10), 178 (1952).

2 Lederberg, J., and St. Clair, J., J. Bact., 75, 143 (1958).

${ }^{3}$ Horoszewicz, A., and Horoszewicz, J., Acta Microbiol. Polonica, 5, 9 (1956).

'Lorkiewicz, Zb., Acta Microbiologica Polonica, 6, 3 (1957).

\section{Somatic Chromosomes of Leptaspis and Streptogyna (Poaceae)}

Вотн Leptaspis and Streptogyna represent very interesting groups from the point of view of the phylogeny of the Poaceae as their external morphology is very primitive. I collected Leptaspis cochleata Thw. and Streptogyna crinita Beauv. in woods at Dulva Kanda (about 4,000 ft.) in Ceylon while I was there in 1957. The root tips of the two species were fixed with Navashin solution at the habitats and, after my return to Japan, dehydrated and embedded in paraffin. Sections were cut at $15 \mu$ and stained by Newton's gentian violet method. Two individuals of $S$. crinita and one individual of $L$. cochleata were used for chromosome observation. As shown in Figs. 1 and 2 , both species had $2 n=24$. The chromosomes of $L$. cochleata are clearly larger than those of $S$. crinita.

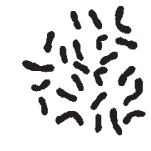

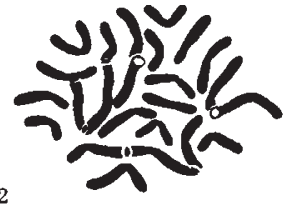

Figs. 1 and 2. Somatic chromosomes. (1) Streptogyna crinita. (2) Leptaspis cochleata $\times 2,000$

Leptaspis is characterized by the diclinous spikelets and belongs to the Olyreae. The tribe, which has never been examined cytologically, is distributed in the tropics, the majority of species being concentrated in Central America and the West Indies. From anatomical investigations of leaf and embryo, as well as morphological studies, the Olyreae are assumed to be distantly related to the Bambuseae. A number of Bambuseae species examined cytologically up to now have had $2 n=48$ or 72 , suggesting the basic chromosome number of 12 , whereas a few have $2 n=36$ or 54. Leptaspis cochleata is similar to the Bambuseae in the basic chromosome number, but the chromosomes of Leptaspis are somewhat larger. 